\title{
Isotropic-nematic phase equilibria of hard-sphere chain fluids-Pure components and binary mixtures
}

\author{
Bernardo Oyarzún, Thijs van Westen, and Thijs J. H. Vlugta) \\ Process and Energy Laboratory, Delft University of Technology, Leeghwaterstraat 39, 2628CB Delft, \\ The Netherlands
}

(Received 15 December 2014; accepted 26 January 2015; published online 11 February 2015)

\begin{abstract}
The isotropic-nematic phase equilibria of linear hard-sphere chains and binary mixtures of them are obtained from Monte Carlo simulations. In addition, the infinite dilution solubility of hard spheres in the coexisting isotropic and nematic phases is determined. Phase equilibria calculations are performed in an expanded formulation of the Gibbs ensemble. This method allows us to carry out an extensive simulation study on the phase equilibria of pure linear chains with a length of 7 to 20 beads (7-mer to 20-mer), and binary mixtures of an 8-mer with a 14-, a 16-, and a 19-mer. The effect of molecular flexibility on the isotropic-nematic phase equilibria is assessed on the 8-mer+19-mer mixture by allowing one and two fully flexible beads at the end of the longest molecule. Results for binary mixtures are compared with the theoretical predictions of van Westen et al. [J. Chem. Phys. 140, 034504 (2014)]. Excellent agreement between theory and simulations is observed. The infinite dilution solubility of hard spheres in the hard-sphere fluids is obtained by the Widom test-particle insertion method. As in our previous work, on pure linear hard-sphere chains [B. Oyarzún, T. van Westen, and T. J. H. Vlugt, J. Chem. Phys. 138, 204905 (2013)], a linear relationship between relative infinite dilution solubility (relative to that of hard spheres in a hard-sphere fluid) and packing fraction is found. It is observed that binary mixtures greatly increase the solubility difference between coexisting isotropic and nematic phases compared to pure components. (C) 2015 AIP Publishing LLC. [http://dx.doi.org/10.1063/1.4907639]
\end{abstract}

\section{INTRODUCTION}

Liquid crystals are elongated molecules characterized by fluid phases with some degree of molecular order. In the isotropic, nematic, and smectic phases, molecules are arranged, respectively, with no positional and no orientational order, with no positional but orientational order, and with positional and orientational order. It has been observed that a certain degree of molecular anisotropy is necessary for the formation of liquid crystal phases. ${ }^{1,2}$ This condition was demonstrated first theoretically by Onsager ${ }^{3}$ for infinitely thin hard rods, and generalized recently by Franco-Melgar et al. ${ }^{4}$ for molecules with different anisotropic shapes. Liquid crystal phases have been observed in molecular simulation studies of anisotropic hard molecules with diverse shapes. ${ }^{5-17}$ From all possible anisotropic systems, tangent hard-sphere chains ${ }^{18-21}$ are the most simple segment-based model of liquid crystals. We are interested in segment-based molecules due to their relevance in the development of physically based perturbation theories describing the behavior of liquid crystals. ${ }^{22,23}$ In this work, we use partially flexible hard-sphere chains formed by a linear part and a fully flexible part for studying the properties of liquid crystal fluids. Partial flexibility is introduced to reproduce the experimental observation that a certain degree of flexibility is required for the stability of liquid crystal phases over the crystal state. ${ }^{2,24}$ Here, flexibility is introduced to study

\footnotetext{
a)Electronic mail: t.j.h.vlugt@ tudelft.nl
}

its effect on the isotropic-nematic phase transition. Molecular simulation results for these systems are, in our knowledge, scarce, principally due to the difficulties on performing phase equilibria calculations of non-simple fluids with classical simulation techniques. In this work, we carry out an extended molecular simulation study on the isotropic-nematic phase equilibria of hard-sphere chain fluids. Furthermore, simulation results are compared with the theoretical predictions obtained from an equation of state recently developed by us. ${ }^{22,23,25}$

In molecular simulations, phase equilibria can be directly obtained from Gibbs ensemble simulations as proposed by Panagiotopoulos et al. ${ }^{26-28}$ In the traditional implementation of this method, chemical potential equilibrium is achieved by transfer of whole molecules between thermally and mechanically equilibrated phases. Molecular transfer between phases is often the rate limiting step in Gibbs ensemble simulations. While transfer of whole molecules between phases is effective only for short and simple molecules, highly anisotropic or complex molecules have a very low probability of transfer acceptance. ${ }^{27,29,30}$ This condition results in poor ergodic sampling within reasonable simulation time. Advanced techniques have been developed to overcome this difficulty. Configurational-bias sampling ${ }^{31,32}$ was implemented in the Gibbs ensemble for improving the simulation of vapor-liquid equilibria of non-simple fluids. ${ }^{33-37}$ Although configurationalbias Monte Carlo shows an improved molecular transfer efficiency over the traditional Gibbs ensemble scheme, it is computational expensive and its efficiency is decreased as 
molecular complexity increases. ${ }^{38-40}$ Even improvements of configurational-bias Monte Carlo as the recoil growth method show to be inefficient for dense systems. ${ }^{41,42}$

Either in the traditional or in the configurational-bias implementation of the Gibbs ensemble, molecular transfers are attempted by insertion/deletion of whole molecules. Intuitively, molecular transfer efficiency can be improved by attempting at every step the transfer of molecular segments rather than of whole molecules. Expanded ensemble techniques are based on this principle. ${ }^{43-45}$ In these methods, the ensemble of a system of whole molecules is expanded into a series of sub-ensembles covering for one fractional molecule, the range between a "ghost" molecule (a molecule without any intermolecular interactions) and a fully coupled molecule (a molecule where all intermolecular interactions are present). In expanded Gibbs ensemble simulations, molecular transfer between phases is achieved by gradually coupling a fractional molecule in one phase while, at the same time, a complementary fractional molecule is decoupled from the other. ${ }^{46,47}$ Gradual coupling/decoupling is performed by a random walk over sub-ensembles. Each one of them corresponding to a defined fractional state that determines the degree of intermolecular coupling of the fractional molecules. In the traditional Metropolis sampling scheme, ${ }^{48}$ a random walk over fractional states results in an uneven distribution of the relative probability of visiting fractional states. This condition restricts molecular transfer between phases, limiting the efficiency of the method. A smooth transition between all fractional states is desired, striving ideally to the same relative probability for visiting any fractional state. For this aim, the partition function of the expanded ensemble is modified by a weight function for each fractional state, changing the Boltzmann statistics of the original system. ${ }^{4-51}$ Numerical values for these weight functions are not known a priori and an iterative method for determining them is required. ${ }^{43-45}$

This paper is organized as follows. In Sec. II, we introduce the expanded Gibbs ensemble simulation method. In Sec. III, simulation results are presented for the isotropic-nematic phase equilibria of pure linear hard-sphere chains (Sec. III A), binary mixtures of linear hard-sphere chains (Sec. III B), binary mixtures of a linear and a partially flexible hard-sphere chains (Sec. III C), and for the infinite dilution solubility of hard spheres in the studied binary mixtures (Sec. III D). Our results are summarized in Sec. IV.

\section{SIMULATION METHODS}

Simulations are performed in an expanded version of the Gibbs ensemble..$^{52}$ The method is based on the gradual exchange of molecules by the coordinated coupling/decoupling of segments of a fractional molecule between phases. There is one fractional molecule present in each phase for each component. Molecules are represented as chain molecules of homonuclear segments made of a linear part and a fully flexible part (only for partially flexible molecules). The fractional molecules are subjected to all Monte Carlo trial moves independent of their fractional state. The simulation method is based on the ideas of Lyubartsev et al. ${ }^{43,53,54}$ on expanded ensembles and is similar in spirit to the continuous fractional component Monte Carlo method of Maginn and coworkers. ${ }^{47,55,56}$ Instead of the continuous insertion presented in their work, here we propose a segment-wise insertion of chain molecules. Similarly, Escobedo and de Pablo developed the expanded ensemble ideas for the calculation of phase equilibria of polymer molecules. ${ }^{45,46,57}$ In their method, fractional molecules in their end-states are counted as whole molecules, and configurational-bias is used for stepwise insertion/deletion of molecular segments. ${ }^{45}$ In the method presented here, the total number of whole molecules remains constant, independent of the fractional state, facilitating the implementation of the method. Furthermore, segments of fractional molecules are sequentially coupled/decoupled avoiding the expensive configurational-bias insertion of segments. In this section, general features of the method are presented, while a detailed description of the coupling parameter trial moves and acceptance rules is presented in the Appendix. The equations shown in this section are general for interacting systems, independent of the specific case of hard-chain fluids studied in this work.

Constant volume expanded Gibbs ensemble simulations are used for determining the phase equilibria of pure components. A constant pressure formulation of the expanded Gibbs ensemble is used for the calculation of the phase equilibria of binary mixtures. Here, we present, for a matter of simplicity, the partition function for systems at constant volume. In the Appendix, the partition function for constant pressure simulations (Eq. (A1)) is shown. The partition function for a multicomponent system made of $n$ components with a total number of $N$ whole molecules and one fractional molecule per component in each subsystem, $a$ and $b$, for a constant total volume $V=V_{a}+V_{b}$, at constant temperature $T$, is given by

$$
\begin{aligned}
Q_{N V T}= & \frac{1}{\Lambda^{3(N+2 n) V}} \prod_{i=1}^{n} \frac{1}{N_{i} !} \sum_{N_{i}^{a}=0}^{N_{i}} \sum_{\lambda_{i}=0}^{m_{i}} \frac{N_{i} !}{N_{i}^{a} ! N_{i}^{b} !} \\
& \times \exp \left[w_{i}\left(\lambda_{i}\right)\right] \int_{0}^{V} d V_{a}\left(V_{a}\right)^{\left(N^{a}+n\right)}\left(V_{b}\right)^{\left(N^{b}+n\right)} \\
& \times \int d \mathbf{s}_{a}^{N^{a}} d \mathbf{s}_{a}^{n} \exp \left(-\frac{U^{a}\left(\mathbf{s}_{a}^{N^{a}}, \mathbf{s}_{a}^{n}, \lambda_{1}, \ldots, \lambda_{n}\right)}{k_{B} T}\right) \\
& \times \int d \mathbf{s}_{b}^{N^{b}} d \mathbf{s}_{b}^{n} \exp \left(-\frac{U^{b}\left(\mathbf{s}_{b}^{N^{b}}, \mathbf{s}_{b}^{n}, \lambda_{1}, \ldots, \lambda_{n}\right)}{k_{B} T}\right),
\end{aligned}
$$

in which $\Lambda$ is the de Broglie wavelength and $k_{B}$ is the Boltzmann factor. $N_{i}=N_{i}^{a}+N_{i}^{b}$ is the total number of whole molecules of component $i$, and $N^{a}=\sum_{i}^{n} N_{i}^{a}$ and $N^{b}=\sum_{i}^{n} N_{i}^{a}$ are the total number of whole molecules in subsystem $a$ and $b$, respectively. A molecule is defined by a chain of interacting segments with a total length $m_{i}$ for a molecule of component $i$. The dimensionless coordinates $\mathbf{s}_{a}^{N^{a}}$ and $\mathbf{s}_{b}^{N^{b}}$ describe the positions of all beads of the $N^{a}$ and $N^{b}$ whole molecules. The dimensionless coordinates $\mathbf{s}_{a}^{n}$ and $\mathbf{s}_{b}^{n}$ describe the positions of all beads of the $n$ fractional molecules in subsystem $a$ and $b$, respectively. Each subsystem has one fractional molecule per component with fractional states defined by the coupling parameter $\lambda_{i}$. The coupling parameter determines the fractional state (number of interacting segments) of the fractional molecules in both subsystems simultaneously, 
i.e., $\lambda_{i}$ coupled segments in subsystem $a$ and $\left(m_{i}-\lambda_{i}\right)$ in subsystem $b$. Therefore, $\lambda_{i}=0$ defines an ideal chain molecule in subsystem $a$ (a molecule where only bonded interactions are present) and a fractional molecule identical (but not equal) to a whole molecule in subsystem $b$. Here, we consider discrete values of the coupling parameter in the range of $\lambda_{i}=\left[0, \ldots, m_{i}\right]$, leading to a total of $m_{i}+1$ possible fractional states. Note that only whole molecules of a specific component are indistinguishable from each other and can be permuted between subsystems. This explains the factors $1 / N_{i} !$ and $N_{i} ! / N_{i}^{a} ! N_{i}^{b}$ ! expressed in terms of the number of whole molecules. The weight functions $w_{i}\left(\lambda_{i}\right)$ are introduced to modify the Boltzmann statistics of the system in order to improve the sampling efficiency of all fractional states. We consider the weight functions of each component to be independent of each other, which is exact in the thermodynamic limit. Weight functions are determined iteratively using the Wang-Landau sampling method ${ }^{58,59}$ as explained below. The total energy of a subsystem $U^{a}$ (the same for subsystem $b$ ) is the sum of the bonded interactions $U^{a \text {,bond }}$ for all molecules in the subsystem, plus the intermolecular $U^{a, \text { inter }}\left(\mathbf{s}_{a}^{N^{a}}, \mathbf{s}_{a}^{n}, \lambda_{1}, \ldots, \lambda_{n}\right)$ and intramolecular interactions $U^{a \text {,intra }}\left(\mathbf{s}_{a}^{N^{a}}, \mathbf{s}_{a}^{n}, \lambda_{1}, \ldots, \lambda_{n}\right)$ for the $N^{a}$ whole and $n$ fractional molecules in the subsystem, $U^{a}=U^{a, \text { non-bonded }}$ $+U^{a \text {, bonded }}=U^{a, \text { inter }}+U^{a, \text { intra }}+U^{a \text {, bond }}$. Only non-bonded interactions (intermolecular and intramolecular) are a function of the fractional state. Bonded interactions do not depend on the fractional state and are equivalent to those of whole molecules.

During a simulation, the following trial moves are attempted: displacements, rotations, reptation, configurationalbias partial regrowths (only for partially flexible molecules), ${ }^{60}$ volume changes, identity exchanges ${ }^{61,62}$ (only for mixtures of linear chains), and coupling parameter changes. They are selected randomly but with a fixed probability proportional to the ratio 100:100:10:100:1:100:1000, respectively. Volume changes are performed isotropically in the logarithm of the volume (in simulations at constant pressure, volume changes are performed in one subsystem at a time). Simulation boxes for a starting isotropic configuration are defined cubic. A rectangular box with edge lengths with a ratio of 1:1.1:1.2 is used for initial nematic configurations. Periodic boundary conditions are used in all simulation boxes. Maximum displacement, rotation, volume, and coupling parameter changes are adjusted for a maximum acceptance ratio of $20 \%$. A Monte Carlo cycle is defined by a number of trial moves equal to the number of molecules in the system, typically in the order of $1 \times 10^{3}$. The number of Monte Carlo cycles required was typically $5 \times 10^{6}$ for equilibration and $2 \times 10^{6}$ cycles for production. Isotropic and nematic phases are identified by the order parameter $S_{2}$ defined by the second-order Legendre polynomial $S_{2}$ $=\frac{1}{N}\left\langle\sum_{i=1}^{N} P_{2}\left(\cos \theta_{i}\right)\right\rangle=\frac{1}{N}\left\langle\sum_{i=1}^{N}\left(\frac{3}{2} \cos ^{2} \theta_{i}-\frac{1}{2}\right)\right\rangle$, where $\theta_{i}$ is the angle between the molecular axis of molecule $i$ and the nematic director. ${ }^{6,21}$ For partially flexible molecules, the molecular axis is defined as the eigenvector corresponding to the smallest eigenvalue of the molecule's moment of inertia tensor. ${ }^{19}$ A value of $S_{2}$ close to 0 identifies the isotropic phase and a value close to 1 is related to the nematic phase. A Markov chain in the space of fractional states can be organized by random changes in the coupling parameter, $\Delta \lambda_{i}$. Two different cases can be identified: changes without molecule transfer and changes with molecule transfer. A coupling parameter change without molecule transfer will occur when the new fractional state $\lambda_{i}^{\text {new }}=\lambda_{i}^{\text {old }}+\Delta \lambda_{i}$ has a value within the range $\left[0, \ldots, m_{i}\right]$ and a change with molecule transfer takes place when $\lambda_{i}^{\text {new }}$ is outside this range. The end-states, $\lambda_{i}=0$ and $\lambda_{i}=m_{i}$, deserve special attention. A fractional molecule with coupling parameter $\lambda_{i}=m_{i}$ is fully coupled to the system; however, this molecule will become equal to a whole one only when a further change in the fractional state takes place (see Fig. 1). A molecule transfer is therefore defined as the state transition $\lambda_{i}=m_{i} \rightarrow 0$ from the old to a new randomly inserted fractional molecule. Strictly, only configurations with fractional molecules in their end-states have a clear physical meaning equivalent to those of a system without fractional molecules. However, sampling only when an end-state is visited has the inconvenience of observables averaged over a reduced number of samples. For a pure component system, this is not truly a limitation, but for multicomponent systems, the probability of visiting an end-state for all components at the same time is reduced to the joint probability of visiting those states. Nevertheless, in the thermodynamic limit, the fractional state does not affect the properties of the system. Moreover, for the finite size systems studied here, we observed that results obtained from end-state sampling do not differ significantly from those obtained from sampling regardless the fractional state. Therefore, we calculate all thermodynamic properties based on the number of whole molecules present in the system independent of the fractional state.

\section{A. Iterative method for determining the weight functions $w(\lambda)$}

Ideally, an equal probability of visiting any fractional state is desired. For this aim, a weight function for every fractional state $w_{i}\left(\lambda_{i}\right)$ is introduced in the partition function (Eq. (1)) to bias the statistics of the system. It is clear that in the modified system, the magnitude of the weight functions has to be inversely proportional to the density of states of the fractional state of the non-modified system. However, the magnitude of these weight functions cannot be known a priori and an iterative method is required for their determination. Wang

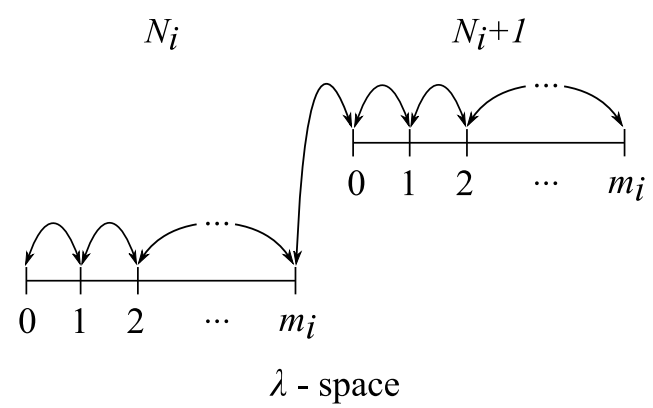

FIG. 1. Schematic representation of changes in the coupling parameter space $\lambda$ for component $i$. When a change in $\lambda_{i}$ reaches the end-state $m_{i}$, the fractional molecule has the same interactions as a whole molecule but it is still not considered as a whole one. A new whole molecule is transferred to the subsystem, $N_{i}+1$, only when a further change in the coupling parameter reaches a state beyond the full fractional state $m_{i}$. 
and Landau proposed an iterative algorithm for estimating the density of states of systems in energy space. ${ }^{58,59}$ Similarly, this algorithm can be used for estimating the density of states in any other parameter space. We use it here for estimating the density of states in the coupling parameter space and therefore determining the values of the weight functions. The method is based on modifying the density of states of the system every time a fractional state is visited to produce a flat histogram. The density of states is changed through the weight function $w_{i}\left(\lambda_{i}\right)$ by a modification factor $v$ that reduces the weight of a fractional state each time it is visited, $w_{i}\left(\lambda_{i}\right) \rightarrow w_{i}\left(\lambda_{i}\right)-v$. When a flat histogram is obtained, the value of the modification factor $v$ is reduced, $v \rightarrow 0.5 \times v$, and the histograms are reseted. A complete flat histogram is not possible and we consider here a flat histogram when the difference between the largest and smallest frequencies is smaller than $10 \%$ of the largest frequency. Changing the density of states at each step alters the Markovian chain and only a modification factor as small as the number precision of the computing machine will strictly obey detailed balance. ${ }^{63}$ This condition is practically impossible and the iteration proceeds until the modification factor is reduced to a very small value. A final value of $1 \times 10^{-9}$ is considered satisfactory in this work. A starting value of $1 \times 10^{-5}$ was sufficient for reaching fast convergence avoiding large oscillations in the value of the weight functions.

\section{B. Infinite dilution solubility of hard spheres}

Infinity dilution solubility of gases is usually expressed in terms of Henry's law constants $H_{k}$. Here, we introduce, for convenience, a dimensionless Henry's law constant defined by $H_{k}^{*}=H_{k} / \rho k_{B} T$, with $\rho$ the number density of molecules. Dimensionless Henry's law constants are related to the infinite dilution residual chemical potential $\mu_{k}^{\text {Res, } \infty}$ of a gas in a solvent by $^{64}$

$$
\ln H_{k}^{*}=\frac{\mu_{k}^{\mathrm{Res}, \infty}}{k_{B} T} .
$$

The infinite dilution residual chemical potential can be obtained by the Widom test-particle insertion method. ${ }^{65}$ For simulations at constant pressure, the chemical potential of hard spheres in a hard-sphere fluid can be calculated by ${ }^{66}$

$$
\frac{\mu_{k}^{\text {Res, } \infty}}{k_{B} T}=-\ln \frac{\langle V p\rangle}{\langle V\rangle} .
$$

The parameter $p$ has a value of either 1 for a random test-particle insertion without overlaps or 0 for an insertion with overlap. In this way, infinite dilution solubility is measured by determining the residual chemical potential of hard spheres in both, the isotropic and the nematic phases at equilibrium. Equilibrium configurations are sampled every $1 \times 10^{3}$ Monte Carlo cycles and a total of 100 test-particle insertions are attempted for each sample. To identify the effect of connectivity and molecular anisotropy on solubility, we define relative Henry's law constants by the ratio between Henry's law constants for the solubility of hard spheres in a hard-sphere chain fluid $H_{k}^{\mathrm{HC}}$ to the solubility of hard spheres in a hard-sphere fluid $H_{k}^{\mathrm{HS}}$,

$$
\frac{H_{k}^{\mathrm{HC}}}{H_{k}^{\mathrm{HS}}}=\exp \left(\frac{\mu_{k, \mathrm{HC}}^{\mathrm{Res}, \infty}}{k_{B} T}-\frac{\mu_{k, \mathrm{HS}}^{\mathrm{Res}, \infty}}{k_{B} T}\right) .
$$

The chemical potential of hard spheres in a hard-sphere fluid is calculated from the Boublik-Mansoori-Carnahan-StarlingLeland equation of state. ${ }^{67}$

\section{SIMULATION RESULTS}

Here, we present simulation results for the isotropicnematic phase equilibria of pure linear hard-sphere chains, binary mixtures of them, and binary mixtures of a linear and a partially flexible hard-sphere chains. Results for the infinite dilution solubility of hard spheres in all studied systems are also reported. Hard-sphere chains are made of segments (beads) of equal diameter $\sigma=1$. All magnitudes are expressed in terms of reduced units with the diameter of a segment $\sigma$ as basis for length. Simulations start with initial isotropic and nematic configurations. Initial configurations are obtained from independent constant pressure simulations $(N P T)$ that approximate the volume of each phase at equilibrium. In the case of pure components, initial estimations of the packing fractions are taken from our previous work. ${ }^{21}$ For the case of mixtures, initial packing fractions, mole fractions, and equilibrium pressures are approximated from the theoretical work of van Westen et al. ${ }^{22}$ The initial size of the simulation boxes is dependent on chain length. The initial edge lengths of both simulation boxes are defined with a value that has to be larger than the length of the longest molecule in the system. Small systems sizes result in simulation boxes with at least one edge length close to the length of the molecule, inducing the formation of smectic phases due to restricted positional order perpendicular to the molecular length. An initial cubic box (isotropic phase) with a size of 25 was sufficient for all studied systems. For the rectangular box (nematic phase), an initial edge length of 25 (the shorter edge) for systems with molecules shorter than 17 beads, and a length of 27 for longer molecules was observed to be sufficient.

\section{A. Linear hard-sphere chain fluids}

The isotropic and nematic phase behaviors of linear hard-sphere chain fluids of different lengths were reported in our previous work. ${ }^{21}$ There, the isotropic and nematic packing fractions at equilibrium were approximated from onephase constant pressure NPT simulations. Here, we obtain the isotropic and nematic packing fractions at equilibrium directly from two-phase simulations. In Table I, the isotropic and nematic packing fractions at equilibrium for linear hard-sphere chains with lengths ranging from 7 to 20 beads are reported. Packing fraction is defined as $\eta=N_{m} v_{m} / V$, where $N_{m}$ is the total number of beads in all whole molecules ( $m$ times $N$ for pure fluids), $v_{m}=\pi \sigma^{3} / 6$ the volume of a single bead, and $V$ the volume of the simulation box. It can be noticed that a maximum in the packing fraction difference exists for the 14mer. However, due to numerical uncertainties, this maximum can be located between the 13-mer and the 17-mer. In our 
TABLE I. Packing fraction of the isotropic phase $\eta^{I}$ and nematic phase $\eta^{N}$, and packing fraction differences $\Delta \eta^{N-I}$ at equilibrium for linear chain molecules with a length $m$ of 7 to 20 beads. Mean values and standard deviations $\sigma$ are obtained from at least 10 independent simulation runs.

\begin{tabular}{lcccccc}
\hline \hline$m$ & $\eta^{I}$ & $\sigma^{I}$ & $\eta^{N}$ & $\sigma^{N}$ & $\Delta \eta^{N-I}$ & $\sigma^{N-I}$ \\
\hline 7 & 0.2945 & 0.0014 & 0.3033 & 0.0010 & 0.0088 & 0.0014 \\
8 & 0.2548 & 0.0011 & 0.2650 & 0.0010 & 0.0102 & 0.0011 \\
9 & 0.2274 & 0.0016 & 0.2391 & 0.0009 & 0.0118 & 0.0016 \\
10 & 0.2066 & 0.0009 & 0.2228 & 0.0007 & 0.0162 & 0.0009 \\
11 & 0.1859 & 0.0010 & 0.2000 & 0.0008 & 0.0141 & 0.0010 \\
12 & 0.1722 & 0.0008 & 0.1903 & 0.0006 & 0.0181 & 0.0008 \\
13 & 0.1636 & 0.0007 & 0.1859 & 0.0006 & 0.0223 & 0.0007 \\
14 & 0.1530 & 0.0009 & 0.1766 & 0.0009 & 0.0236 & 0.0009 \\
15 & 0.1426 & 0.0011 & 0.1657 & 0.0011 & 0.0231 & 0.0011 \\
16 & 0.1344 & 0.0008 & 0.1574 & 0.0013 & 0.0230 & 0.0013 \\
17 & 0.1268 & 0.0005 & 0.1497 & 0.0009 & 0.0230 & 0.0009 \\
18 & 0.1192 & 0.0004 & 0.1401 & 0.0006 & 0.0209 & 0.0006 \\
19 & 0.1121 & 0.0005 & 0.1311 & 0.0008 & 0.0190 & 0.0008 \\
20 & 0.1072 & 0.0008 & 0.1229 & 0.0010 & 0.0157 & 0.0010 \\
\hline \hline
\end{tabular}

previous work, ${ }^{21}$ we located this maximum for a 15 -mer but without data for the 16-mer to the 19-mer. A large difference in the packing fraction between the isotropic and the nematic phases is relevant for the solubility difference of hard spheres between both phases, as shown in Sec. III D.

\section{B. Binary mixtures of linear hard-sphere chain fluids}

In this section, we present results for the isotropic-nematic phase equilibria of binary mixtures of linear hard-sphere chains. Figs. 2, 3, and 4 show the reduced pressure $P^{*}$ and packing fraction $\eta v s$. mole fraction of the largest component $x_{2}$ for mixtures of an 8-mer with a 14-, a 16-, and a 19-mer, respectively. We use the plus sign to refer to a mixture, e.g., 8mer + 14-mer indicates a binary mixture of an 8-mer with a 14mer. Reduced pressures are defined relative to the molecular volume of the shortest component (in all cases, the 8-mer) by $P^{*}=P v_{8-\text { mer }} / k_{B} T$, where $P$ is the pressure of the system, and $v_{8-\text { mer }}$ is the molecular volume of an 8-mer. Simulation results are compared to theoretical predictions obtained from a Vega-Lago rescaled Onsager theory by van Westen $\mathrm{et} \mathrm{al.}{ }^{23}$ For the larger part of the phase diagrams, excellent agreement between theory and simulations is obtained; however, for systems very rich in the short component $\left(x_{2} \approx 0\right)$, a small overestimation of pressure by the theory is observed. The offset is a consequence of approximations of higher virial coefficients (which are treated by a Vega-Lago rescaling procedure). For smaller chain lengths, the phase transition is shifted to higher packing fractions. Therefore, any errors introduced by the approximate treatment of the higher virial coefficients become apparent, leading to somewhat larger deviations between theory and simulations. ${ }^{22,23}$ To the best of our knowledge, the only available simulation data for the phase equilibria of mixtures of linear hard-sphere chains are those of Escobedo and de Pablo. ${ }^{68}$ In Fig. 3, the simulation results of Escobedo and de Pablo are compared with ours and with the theoretical predictions of van Westen et al. ${ }^{23}$ All results are in good agreement with each other, validating previous results and our own simulation technique.

For all systems studied, a phase split into an isotropic and a nematic phase is observed. This phase split is accompanied by a fractionation of the mixture into an isotropic phase richer in the short component and a nematic phase richer in the long component. Phase split and fractionation occur as a consequence of maximizing the total entropy of the system, balancing orientational, translational, and mixing entropy. In hard systems, this entropy maximum is associated to a maximization of the free volume or equivalently to a minimization of the excluded volume. ${ }^{69,70}$ For a mixture with a specific concentration, at pressures below the isotropicnematic region, orientational and mixing entropies dominate and a one-phase isotropic system is observed. At higher pressures, the translational entropy of the isotropic phase is reduced, and a further gain in total entropy is reached by phase split of the system into a nematic and an isotropic phase. The loss in orientational entropy due to the phase split and the loss of mixing entropy by the accompanying fractionation are more than compensated by the gain in translational entropy. Phase split from an isotropic to a nematic phase increases the translational entropy as a consequence of a reduced excluded volume in the nematic phase when chains lay fairly parallel. ${ }^{3,4,71}$ Fractionation occurs due to a larger tendency to align of the long chains compared to the short chains. ${ }^{72,73}$ This tendency is a consequence of a larger excluded volume difference between the isotropic and nematic phases for the long chains than for the short chains. ${ }^{74}$ This excluded volume difference increases with chain length, broadening the fractionation of the system into a nematic phase richer in long chains and an isotropic phase more
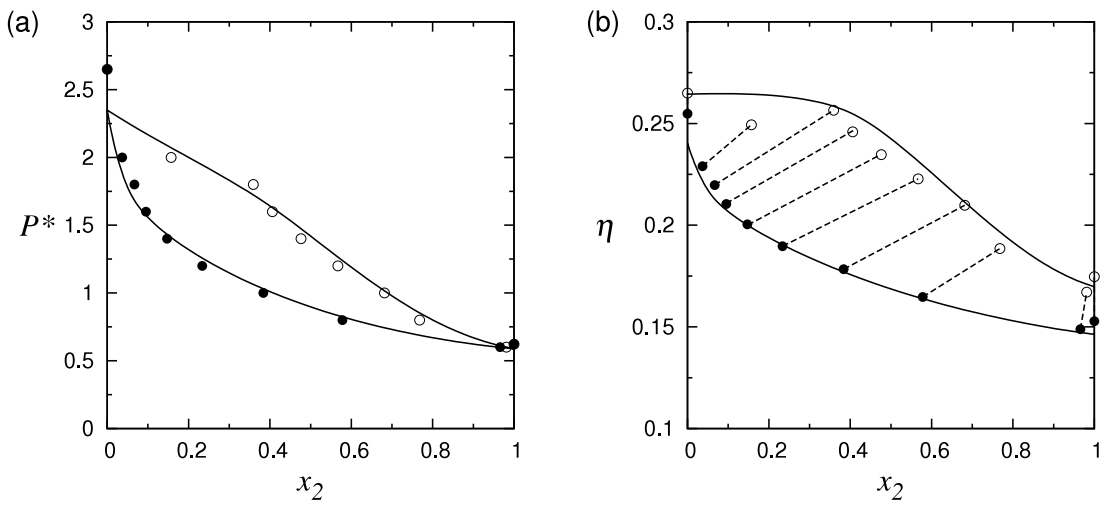

FIG. 2. Isotropic-nematic phase equilibria for the binary mixture 8-mer +14 -mer. (a) Reduced pressure $P^{*} v s$. mole fraction of the largest component $x_{2}$, (b) packing fraction $\eta v s$. mole fraction of the largest component $x_{2}$. Black $(\bullet)$ and empty (o) dots are simulation results for the isotropic phase and nematic phase, respectively. Broken lines are constant pressure tie-lines. Solid lines are theoretical results obtained from a rescaled Onsager theory by van Westen et al. $^{23}$ 
(a)

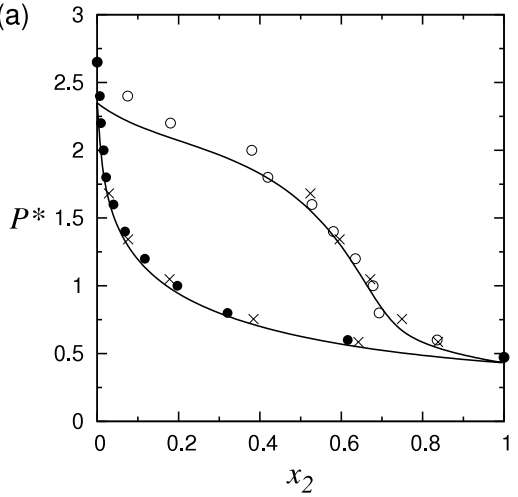

(b)

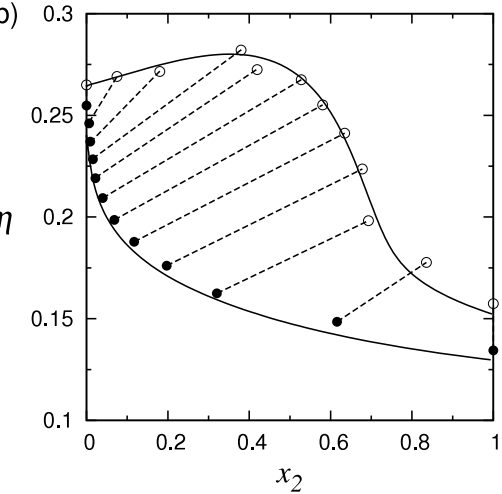

FIG. 3. Isotropic-nematic phase equilibria for the binary mixture 8-mer +16 -mer. (a) Reduced pressure $P^{*} v s$. mole fraction of the largest component $x_{2}$, (b) packing fraction $\eta v s$. mole fraction of the largest component $x_{2}$. Symbols and lines as in Fig. 2. Crosses $(x)$ are simulation results from Escobedo and de Pablo. ${ }^{68}$ depleted of them. Increasing fractionation with the difference in molecular length was reported in previous studies $23,75,76$ and is also observed in our results. Figs. 2-4(a) show a broader isotropic-nematic region, in binary mixtures, as the length of the long chain (14-, 16-, 19-mer) increases for a constant length of the short chain (8-mer). The behavior of packing fraction with mole fraction of the largest component is shown in Figs. 2-4(b). It can be observed that the packing fraction of the isotropic phase at equilibrium decreases rapidly with mole fraction. This decrement is caused by the alignment potential that long chains introduce in the fluid, facilitating the formation of the nematic phase at lower equilibrium pressures. In the nematic phase, at low concentrations of the long chains, the packing fraction remains fairly constant (Fig. 2) or increases with mole fraction (Figs. 3 and 4), although the coexistence pressure decreases. This behavior is a consequence of the "higher packing" introduced by the connectivity of spheres in the long chain. At larger concentrations, the lower isotropicnematic equilibrium pressures effectively reduce the packing fraction of the nematic phase. A maximum in the packing fraction difference between the isotropic and nematic phases is observed for all systems, which increases with the difference in chain length between the long and the short chains.

In the theoretical study of van Westen et al., a nematicnematic region was detected for binary mixtures at high reduced pressures. Specifically, it was shown that for the mixture 8-mer + 19-mer, a nematic-nematic region follows the isotropic-nematic equilibria after a triple point at a reduced pressure of 2.234. ${ }^{23}$ Here, we perform phase equilibria simulations for the 8-mer + 19-mer mixture at high pressures to try to disclose the existence of the nematic-nematic region.
Simulations at a reduced pressure of 2.4 show phase equilibrium between a nematic phase, concentrated in the short chains, and a smectic-A phase, concentrated in the long chains. The smectic phase is formed by a layer of short chain molecules without clear positional order between two layers of long chain molecules with defined positional order. At a higher pressure of 3.0, the nematic phase is turned into a phase where long chains are locally clustered with a defined orientation and position surrounded by short chains oriented towards the nematic director but with no clear positional order. At this pressure, the smectic-A phase is clearly defined arranging a layer of short chains, formed by two consecutive layers of short chains, between two layers of long chains. We consider these results as preliminary since the systems and corresponding box sizes are too small to accommodate smectic phases without any influence of the periodic boundary conditions on the positional order of the system. Nevertheless, the theoretical existence of nematic and smectic phases in equilibrium at high pressures has been reported for the case of hard-spherocylinders. ${ }^{77-79}$ And, although, the theoretical results of van Westen et al. do not consider the formation of smectic phases, Cinacchi et al. ${ }^{80}$ showed that a metastable nematic-nematic region can precede the formation of stable nematic-smectic equilibria.

\section{Binary mixtures of a linear and a partially flexible hard-sphere chain fluids}

Partially flexible molecules are introduced to study the effect of molecular flexibility on the isotropic-nematic phase equilibria. A partially flexible molecule is defined as a hardsphere chain molecule with a linear part and a fully flexible
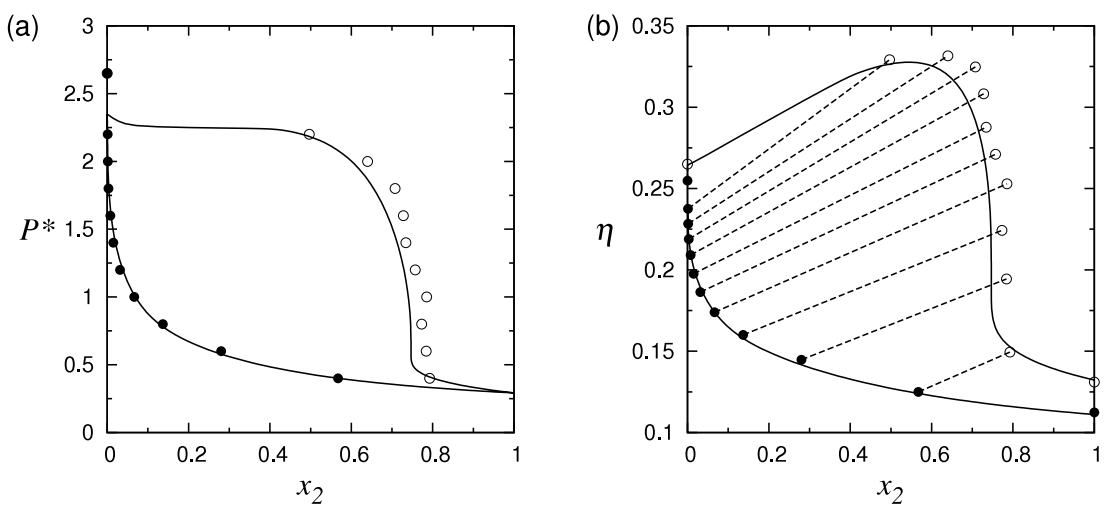

FIG. 4. Isotropic-nematic phase equilibria for the binary mixture 8 -mer +19 -mer. (a) Reduced pressure $P^{*} v s$. mole fraction of the largest component $x_{2}$, (b) packing fraction $\eta v s$. mole fraction of the largest component $x_{2}$. Symbols and lines as in Fig. 2. 

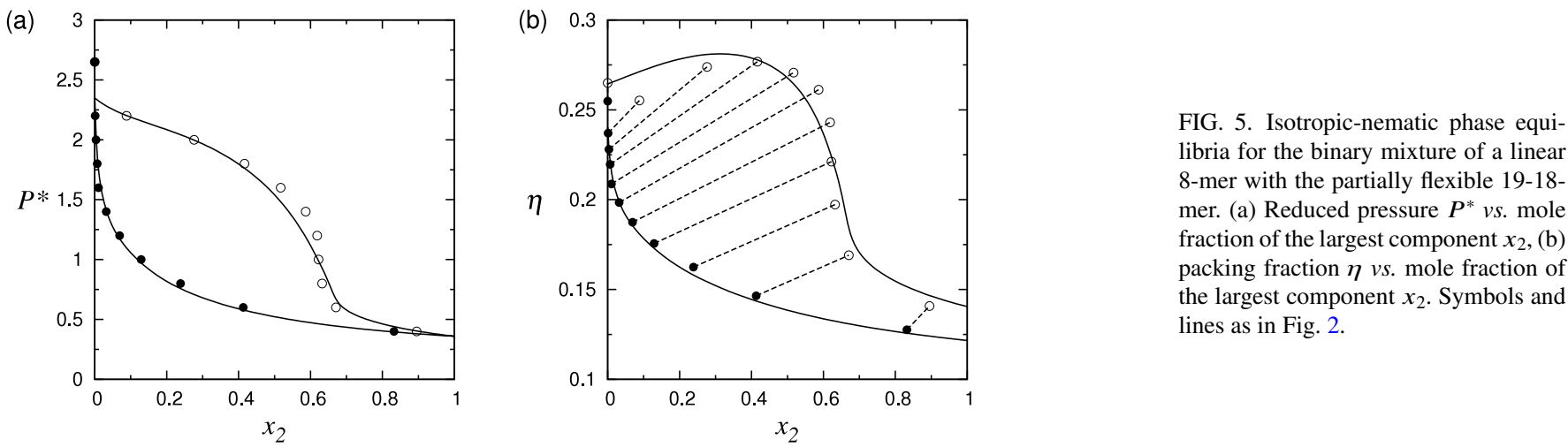

part. We employ the notation 19-18-mer for a chain molecule with a total length of 19 beads formed by a linear part of 18 beads and a fully flexible part of 1 bead. The fully flexible part is freely to move around the geometrical center of the previous segment, avoiding overlaps with other molecules and with the molecule itself. In this work, we investigate the effect of flexibility in two binary mixtures: a linear 8mer with a 19-18-mer and a linear 8-mer with a 19-17-mer. Figs. 5 and 6 show the results for the isotropic-nematic phase equilibria in these systems. Comparing these results with the fully linear case, Fig. 4, it is observed that flexibility has the effect of, first, increasing the pressure at which the two-phase region starts to appear, and second, reducing the degree of fractionation between coexisting phases. Flexibility decreases the anisotropy of the chain, diminishing the gain in translational entropy that can be obtained from a phase split into a nematic phase. Therefore, a closer packing is needed for the nematic phase to start to form, increasing the pressure at which the isotropic-nematic equilibria take place. The reduced fractionation is explained by a lower excluded volume difference between the isotropic and the nematic phases (lower tendency to align) for the partially flexible chain compared to the linear case, as shown for the pair excluded volume of two partially flexible molecules by van Westen et al. ${ }^{81}$

\section{Infinite dilution solubility of hard spheres}

In our previous work for pure components, ${ }^{21}$ we showed that a linear relationship exists between relative infinite dilution solubility, defined as Eq. (4), and packing fraction. It was found that this relationship is practically independent of chain length for chains of 10 beads and longer. Moreover, it was demonstrated that this relationship does not depend on the liquid crystal state of the fluid, either isotropic or nematic, being only a function of packing fraction. In principle, the independence of relative solubility on chain length for long chains (longer than 10 beads) can be explained from pair excluded volume interactions. van Westen et al. ${ }^{81}$ derived an expression for the dimensionless pair excluded volume between two linear chains of different length $V_{\mathrm{ex}}^{*}=V_{\mathrm{ex}} / V_{\bar{m}}$, where $V_{\text {ex }}$ is the pair excluded volume and $V_{\bar{m}}$ is the volume of a chain of $\bar{m}$ hard spheres. Here, $\bar{m}=\left(m_{1}+m_{2}\right) / 2$, with $m_{1}$ and $m_{2}$ as the lengths of the two linear chains. If one of the chains is considered just as a hard sphere, that expression reduces to $V_{\mathrm{ex}}^{*}=(11 \bar{m}-3) / \bar{m}$. It can be observed that as $\bar{m}$ increases, the value of the excluded volume approaches a limiting value. For chains of 10 beads and longer, changes in the relative excluded volume are progressively smaller, explaining in principle the unnoticeable effect of chain length on solubility reported in our previous work. It is remarkable that this argument taken from pair molecular interactions also holds for high packing fractions where multi-body interactions start to be relevant.

Fig. 7 shows the relative infinite dilution solubility of hard spheres in binary mixtures of linear, and linear with partially flexible hard-sphere chains vs. packing fraction. It can be observed that, as for the pure component case (Ref. 21), a linear relationship of relative solubility with packing fraction independent of the mixture type is also obtained. Fig. 7 includes a linear regression for all reported data. The slope and intercept of this regression are, respectively, $-2.674 \pm 0.086$ and $1.065 \pm 0.015$, which are equivalent to the ones reported in our previous work. This result is coherent with the results of solubility in pure components, where for relatively long enough chains, solubility seems to be independent of
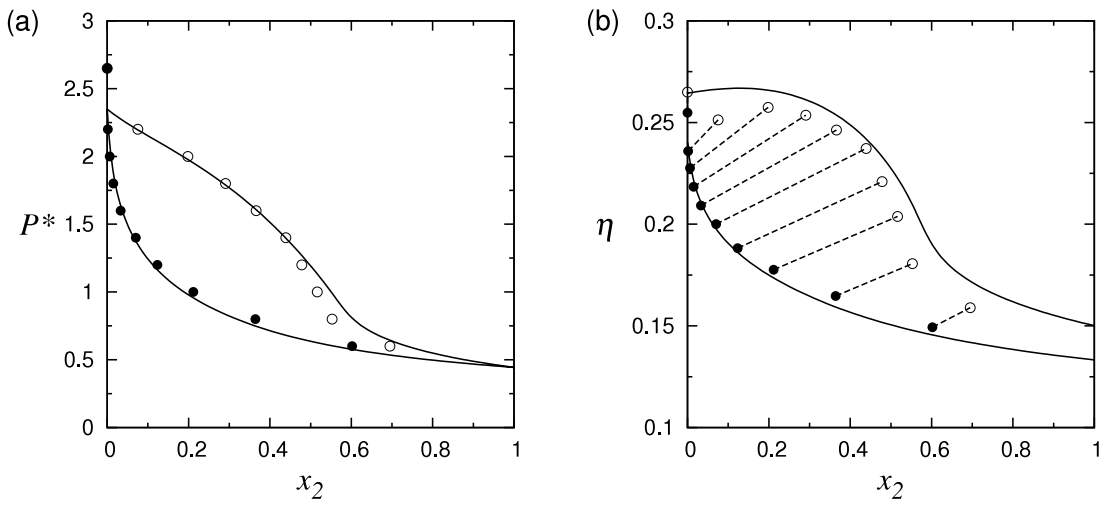

FIG. 6. Isotropic-nematic phase equilibria for the binary mixture of a linear 8-mer with the partially flexible 19-17mer. (a) Reduced pressure $P^{*} v s$. mole fraction of the largest component $x_{2}$, (b) packing fraction $\eta v s$. mole fraction of the largest component $x_{2}$. Symbols and lines as in Fig. 2. 


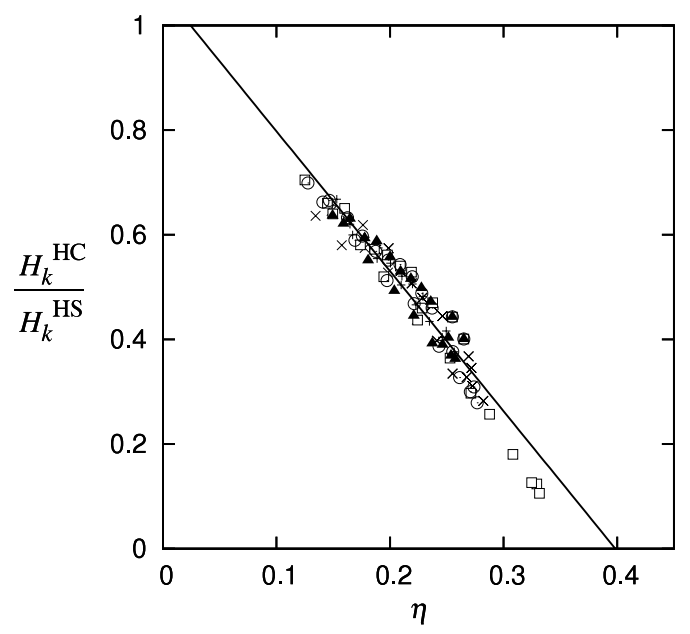

FIG. 7. Relative infinite dilution solubility $H_{k}^{\mathrm{HC}} / H_{k}^{\mathrm{HS}} v s$. packing fraction $\eta$ for hard spheres in binary mixtures of linear and partially flexible hard-sphere chains in the isotropic and nematic phases at equilibrium. (+) 8-mer + 14-mer, (×) 8-mer + 16-mer, (ㅁ) 8-mer + 19-mer, (\) 8-mer + 19-18-mer, and (o) 8 -mer $+19-17$-mer. The solid line is a linear regression calculated from all data.

chain length. It has to be noticed that similarly to the pure component case, this linear relationship would eventually not be independent on chain length if one of the components forming the mixture becomes very small.

Finally, Fig. 8 shows dimensionless Henry's law constants, defined by Eq. (2), vs. mole fraction of long chains for the solubility of hard spheres in a mixture of linear chains, 8-mer + 16-mer, at the isotropic-nematic coexistence. It can be observed that the solubility of hard spheres in the isotropic phase increases with mole fraction (indicated by a decrease in Henry's law constants), showing a rapid increment at mole fractions close to the pure short chain fluid. The solubility in the nematic phase is fairly decreased at low values of the mole fraction showing a minimum after which it increases monotonically. This behavior is analogous to the changes in packing fraction observed in Fig. 3. A maximum in the solubility difference between the isotropic and the nematic phases is detected at a mole fraction of

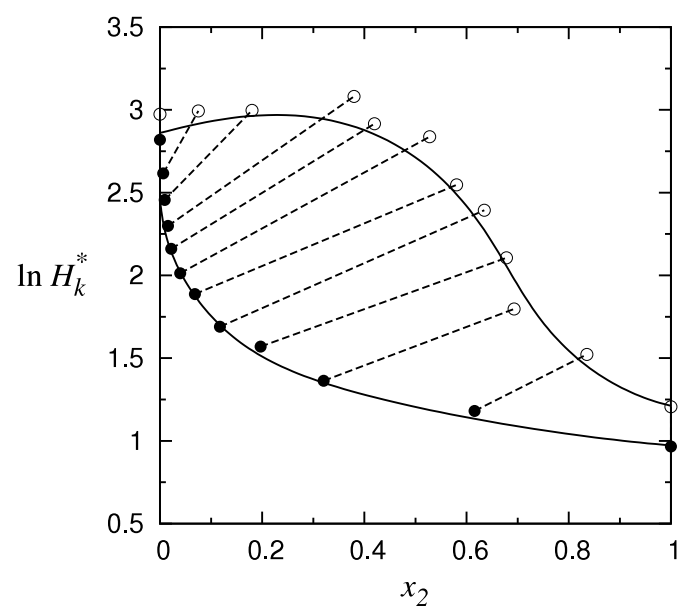

FIG. 8. Dimensionless Henry's law constants $\ln H_{k}^{*} v s$. mole fraction of the longest component $x_{2}$ for the binary mixture 8-mer +16 -mer in the isotropic and nematic phases at equilibrium. Symbols and lines as in Fig. 2. around 0.5 , corresponding to the maximum in the packing fraction difference between both phases observed at the same concentration.

\section{CONCLUSIONS}

The isotropic-nematic phase equilibria of pure components and binary mixtures of linear and partially flexible hardsphere chains were directly calculated from expanded Gibbs ensemble simulations. For pure components, the packing fractions of the isotropic and nematic phases at equilibrium were obtained for linear chains with a length of 7 to 20 beads. These results show a maximum in the packing fraction difference between both phases for a chain length between the 13-mer and the 17-mer. For binary mixtures, the packing fraction and mole fraction of the coexisting isotropic and nematic phases were obtained for mixtures of a linear 8-mer with a 14-, 16-, and 19-mer. Phase split, between an isotropic and a nematic phases, and fractionation, between an isotropic phase richer in the short component and a nematic phase richer in the large component, are observed for all binary mixtures. The degree of fractionation between both phases increases with the chain length of the largest component for a constant length of the short chain. The effect of molecular flexibility was studied in binary mixtures of an 8-mer with a partially flexible 19-18-mer and a 19-17-mer. Flexibility increases the isotropic-nematic equilibrium pressure and reduces the degree of fractionation between both phases. The relative infinite dilution solubility of hard spheres in the isotropic and nematic phases at equilibrium was obtained for all studied binary mixtures. A linear relationship between relative solubility and packing fraction is found. This linear relationship is equivalent to the one obtained in our previous work for pure linear hard-sphere chains. ${ }^{21}$ Binary mixtures show a larger packing fraction difference and, therefore, a larger hard sphere solubility difference between the isotropic and the nematic phases than the constituent pure components. This result shows that mixtures of liquid crystals have the potential of largely increasing the solubility difference of gases between the isotropic and the nematic phases. A large solubility difference is relevant for the use of liquid crystals as solvents for gas separation applications. Recently, de Loos et al. showed experimentally that liquid crystals can be used as new solvents for $\mathrm{CO}_{2}$ capture. ${ }^{82-84} \mathrm{In}$ line with the findings obtained in this work, experimental results show that binary mixtures of liquid crystals can provide a larger $\mathrm{CO}_{2}$ solubility difference between the isotropic and the nematic phases than the pure components. ${ }^{84}$

\section{ACKNOWLEDGMENTS}

This research is supported by the Stichting voor Technische Wetenschappen (Dutch Technology Foundation, STW), applied science division of the Nederlandse Organisatie voor Wetenschappelijk Onderzoek (Netherlands Organization for Scientific Research, NWO), and the Technology Program of the Ministry of Economic Affairs. In addition, this work was sponsored by the Stichting Nationale Computerfaciliteiten 
(National Computing Facilities Foundation, NCF) for the use of supercomputing facilities, with financial support from NWO (Grant No. MP-213-14).

\section{APPENDIX: SIMULATION DETAILS}

\section{Partition function NPT simulations}

The partition function for constant pressure NPT expanded Gibbs ensemble simulations is given by (see Ref. 85 for constant pressure Gibbs ensemble)

$$
\begin{aligned}
Q_{N P T}= & \left(\frac{P}{k_{B} T}\right)^{2} \frac{1}{\Lambda^{3(N+2 n)}} \prod_{i=1}^{n} \frac{1}{N_{i} !} \sum_{N_{i}^{a}=0}^{N_{i}} \sum_{\lambda_{i}=0}^{m_{i}} \frac{N_{i} !}{N_{i}^{a} ! N_{i}^{b} !} \\
& \times \exp \left[w_{i}\left(\lambda_{i}\right)\right] \int_{0}^{\infty} d V_{a} \exp \left(-\frac{P V_{a}}{k_{B} T}\right)\left(V_{a}\right)^{\left(N^{a}+n\right)} \\
& \times \int_{0}^{\infty} d V_{b} \exp \left(-\frac{P V_{b}}{k_{B} T}\right)\left(V_{b}\right)^{\left(N^{b}+n\right)} \\
& \times \int d \mathbf{s}_{a}^{N^{a}} d \mathbf{s}_{a}^{n} \exp \left(-\frac{U^{a}\left(\mathbf{s}_{a}^{N^{a}}, \mathbf{s}_{a}^{n}, \lambda_{1}, \ldots, \lambda_{n}\right)}{k_{B} T}\right) \\
& \times \int d \mathbf{s}_{b}^{N^{b}} d \mathbf{s}_{b}^{n} \exp \left(-\frac{U^{b}\left(\mathbf{s}_{b}^{N^{b}}, \mathbf{s}_{b}^{n}, \lambda_{1}, \ldots, \lambda_{n}\right)}{k_{B} T}\right),
\end{aligned}
$$

symbols are described in the main part of the text. The constant value $\left(P / k_{B} T\right)^{2}$ is introduced to keep the partition function dimensionless. ${ }^{60}$

\section{Coupling parameter trial change}

In this section, we provide an extended description and derivation of the acceptance rules for changes in the coupling parameter. The notation used in deriving the acceptance rules is taken from the work of Rull et al. ${ }^{86}$ Changes in the coupling parameter are performed for one component, chosen randomly, at a time. After the component is selected, the subsystem, where an increase in the fractional state is attempted, is selected randomly with same probability for both subsystems. After this, a coupling parameter change for the selected component $\Delta \lambda_{i}$ is chosen randomly from the $m_{i}+1$ possible fractional states. As shown below, the probability of accepting a change in the coupling parameter is different for the case that a change with or without molecular transfer is attempted. If for component $i$, the subsystem does not have any whole molecules, a coupling parameter change for this component is automatically rejected if a fractional change with molecular transfer is attempted. Every attempt of changing the configuration of the system from $\alpha$ to $\beta$ has to satisfy the condition of microscopic reversibility, i.e., the probability of the forward $\mathcal{P}_{\alpha \beta}$ and backwards $\mathcal{P}_{\beta \alpha}$ steps has to be equal

$$
\mathcal{P}_{\alpha \beta}=\mathcal{P}_{\beta \alpha}
$$

Here, we develop the condition of microscopic reversibility for a change in the coupling parameter of component $i$ with and without molecular transfer.

\section{a. Coupling parameter change without molecular transfer}

For a change in the coupling parameter without molecular transfer $\lambda_{i}^{\text {new }}=\lambda_{i}^{\text {old }}+\Delta \lambda_{i}$, the condition of microscopic reversibility (Eq. (A2)) is given by

$$
\mathcal{P}_{\alpha} \mathcal{P}^{\text {box }, a} \mathcal{P}^{\text {comp }, i} \mathcal{P}_{\alpha \beta}^{\text {acc }}=\mathcal{P}_{\beta} \mathcal{P}^{\text {box }, b} \mathcal{P}^{\text {comp }, i} \mathcal{P}_{\beta \alpha}^{\text {acc }},
$$

where

$\mathcal{P}_{\alpha} \quad$ : probability of the system to be in configuration $\alpha$

$\mathcal{P}^{\text {box }, a} \quad$ : probability of selecting subsystem $a$ for an increase in the fractional state

$\mathcal{P}^{\text {comp }, i}$ : probability of selecting component $i$ for a coupling parameter change

$\mathcal{P}_{\alpha \beta}^{\text {acc }} \quad$ : acceptance probability for a configurational change from $\alpha$ to $\beta$.

The probability $\mathcal{P}_{\alpha}$ for the system to be in configuration $\alpha$ is proportional to the pseudo-Boltzmann factor given by (see Eq. (1))

$$
\begin{aligned}
\mathcal{P}_{\alpha} \propto & \exp \left[\sum_{i=1}^{n}\left(\ln \left(\frac{N_{i} !}{N_{i}^{a} ! N_{i}^{b} !}\right)+w_{i}\left(\lambda_{i}\right)\right)\right. \\
& +\left(N^{a}+n\right) \ln V_{a}+\left(N^{b}+n\right) \ln V_{b} \\
& \left.-\frac{U^{a}\left(\mathbf{s}_{a}^{N^{a}}, \mathbf{s}_{a}^{n}, \lambda_{1}, \ldots, \lambda_{n}\right)}{k_{B} T}-\frac{U^{b}\left(\mathbf{s}_{b}^{N^{b}}, \mathbf{s}_{b}^{n}, \lambda_{1}, \ldots, \lambda_{n}\right)}{k_{B} T}\right] .
\end{aligned}
$$

The probability of selecting any of both subsystems for an increase in the fractional state is defined as $\mathcal{P}^{\text {box }, a}=\mathcal{P}^{\text {box }, b}$ $=1 / 2$. The probability of selecting component $i$ for a coupling parameter change $\mathcal{P}^{\text {comp, } i}$ is independent of the configuration of the system. Therefore, the criterion for a reversible change in $\lambda_{i}$ without molecular transfer is given by

$$
\begin{aligned}
\mathcal{P}^{\Delta \lambda_{i}}= & \frac{\mathcal{P}_{\alpha \beta}^{\text {acc }}}{\mathcal{P}_{\beta \alpha}^{\text {acc }}} \\
= & \frac{\mathcal{P}_{\beta}}{\mathcal{P}_{\alpha}} \\
= & \exp \left[w_{i}\left(\lambda_{i}^{\text {new }}\right)-w_{i}\left(\lambda_{i}^{\text {old }}\right)\right. \\
& -\left(\frac{U^{a}\left(\mathbf{s}_{a}^{N^{a}}, \mathbf{s}_{a}^{n}, \lambda_{i}^{\prime \text { new }}\right)}{k_{B} T}-\frac{U^{a}\left(\mathbf{s}_{a}^{N^{a}}, \mathbf{s}_{a}^{n}, \lambda_{i}^{\text {old }}\right)}{k_{B} T}\right) \\
& \left.-\left(\frac{U^{a}\left(\mathbf{s}_{b}^{N^{b}}, \mathbf{s}_{b}^{n}, \lambda_{i}^{\text {new }}\right)}{k_{B} T}-\frac{U^{b}\left(\mathbf{s}_{b}^{N^{b}}, \mathbf{s}_{b}^{n}, \lambda_{i}^{\prime \text { old }}\right)}{k_{B} T}\right)\right],
\end{aligned}
$$

where $\lambda_{i}^{\text {new }}$ and $\lambda_{i}^{\text {'old }}$ indicate that only the coupling parameter of component $i$ is changed. A new configuration $\beta$ is accepted with a probability given by $\min \left(1, \mathcal{P}^{\Delta \lambda_{i}}\right)$.

\section{b. Coupling parameter change with molecular transfer}

For a change in the configuration of the system from $\alpha$ to $\beta$ by a change in the coupling parameter for component $i$ with molecular transfer, from subsystem $a$ to $b$, the condition 
of microscopic reversibility is given by

$$
\begin{aligned}
& N_{i}^{a} ! N_{i}^{b} ! \mathcal{P}_{\alpha} \mathcal{P}^{\text {box }, a} \mathcal{P}^{\text {comp }, i} \mathcal{P}_{\alpha}^{\text {molecule }, a} \mathcal{P}_{\alpha \beta}^{\text {acc }} \\
& \quad=\left(N_{i}^{a}-1\right) !\left(N_{i}^{b}+1\right) ! \mathcal{P}_{\beta} \mathcal{P}^{\text {box }, b} \mathcal{P}^{\text {comp }, i} \mathcal{P}_{\beta}^{\text {molecule }, b} \mathcal{P}_{\beta \alpha}^{\text {acc }},
\end{aligned}
$$

where

$$
\begin{aligned}
& \mathcal{P}_{\alpha} \quad \text { : probability of the system to be in configuration } \\
& \alpha \\
& \mathcal{P}^{\text {box }, a} \quad \text { : probability of selecting subsystem } a \text { for an } \\
& \text { increase in the fractional state }
\end{aligned}
$$

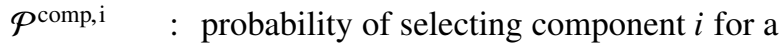

$$
\begin{aligned}
& \text { coupling parameter change } \\
& \mathcal{P}_{\alpha \beta}^{\text {acc }} \quad: \text { acceptance probability for a configurational } \\
& \text { change from } \alpha \text { to } \beta \\
& \mathcal{P}_{\alpha}^{\text {molecule, } a} \text { : probability of choosing a molecule in } \\
& \text { subsystem } a \text { as the new fractional molecule, } \\
& 1 / N_{i}^{a} \\
& \mathcal{P}_{\beta}^{\text {molecule, } b} \text { : probability of choosing a molecule in } \\
& \text { subsystem } b \text { as the new fractional molecule, } \\
& 1 /\left(N_{i}^{b}+1\right) \text {. }
\end{aligned}
$$

The factors $N_{i}^{a} ! N_{i}^{b}$ and $\left(N_{i}^{a}-1\right) !\left(N_{i}^{b}+1\right)$ ! represent all possible permutations of selecting a molecule of component $i$ in the system before and after molecular transfer takes place. In this case, the probability that the system is in configuration $\beta$, with a molecule of component $i$ transferred from $a$ to $b$, is proportional to

$$
\begin{aligned}
\mathcal{P}_{\beta} \propto & \exp \left[\sum_{j \neq i}^{n}\left(\ln \left(\frac{N_{j} !}{N_{j}^{a} ! N_{j}^{b} !}\right)+w_{j}\left(\lambda_{j}\right)\right)\right. \\
+ & \ln \left(\frac{N_{i} !}{\left(N_{i}^{a}-1\right) !\left(N_{i}^{b}+1\right) !}\right)+w_{i}\left(\lambda_{i}\right) \\
+ & \left(N^{a}+n-1\right) \ln V_{a}+\left(N^{b}+n+1\right) \ln V_{b} \\
& -\frac{U^{a}\left(\mathbf{s}_{a}^{N^{a}-1}, \mathbf{s}_{a}^{n}, \lambda_{1}, \ldots, \lambda_{n}\right)}{k_{B} T} \\
& \left.-\frac{U^{b}\left(\mathbf{s}_{b}^{N^{b}+1}, \mathbf{s}_{b}^{n}, \lambda_{1}, \ldots, \lambda_{n}\right)}{k_{B} T}\right] .
\end{aligned}
$$

Therefore, the criterion of a reversible change in $\lambda_{i}$ with molecular transfer for component $i$ is given by

$$
\begin{aligned}
\mathcal{P}_{\mathrm{tr}}^{\Delta \lambda_{i}}= & \frac{\mathcal{P}_{\alpha \beta}^{\mathrm{acc}}}{\mathcal{P}_{\beta \alpha}^{\mathrm{acc}}} \\
= & \frac{\left(N_{i}^{a}-1\right) !\left(N_{i}^{b}+1\right) !}{N_{i}^{a} ! N_{i}^{b} !} \frac{\mathcal{P}_{\beta}^{\text {molecule }, b}}{\mathcal{P}_{\alpha}^{\text {molecule }, a}} \frac{\mathcal{P}_{\beta}}{\mathcal{P}_{\alpha}} \\
= & \exp \left[\ln \left(\frac{N_{i}^{a}}{N_{i}^{b}+1} \frac{V_{b}}{V_{a}}\right)+w_{i}\left(\lambda_{i}^{\text {new }}\right)-w_{i}\left(\lambda_{i}^{\text {old }}\right)\right. \\
& -\left(\frac{U^{a}\left(\mathbf{s}_{a}^{N^{a}-1}, \mathbf{s}_{a}^{n}, \lambda_{i}^{\text {new }}\right)}{k_{B} T}-\frac{U^{a}\left(\mathbf{s}_{a}^{N^{a}}, \mathbf{s}_{a}^{n}, \lambda_{i}^{\text {old }}\right)}{k_{B} T}\right) \\
& \left.-\left(\frac{U^{b}\left(\mathbf{s}_{b}^{N^{b}+1}, \mathbf{s}_{a}^{n}, \lambda_{i}^{\prime \text { new }}\right)}{k_{B} T}-\frac{U^{b}\left(\mathbf{s}_{b}^{N^{b}}, \mathbf{s}_{a}^{n}, \lambda_{i}^{\prime \text { old }}\right)}{k_{B} T}\right)\right],
\end{aligned}
$$

where the new fractional state is equal to $\lambda_{i}^{\text {new }}=\lambda_{i}^{\text {old }}+\Delta \lambda_{i}$ $-\left(m_{i}+1\right)$. Here, again $\lambda_{i}^{\text {new }}$ and $\lambda_{i}^{\text {old }}$ indicate that only the coupling parameter of component $i$ is changed. A new configuration $\beta$ is accepted with a probability given by $\min \left(1, \mathcal{P}_{\mathrm{tr}}^{\Delta \lambda_{i}}\right)$.

${ }^{1}$ P. G. de Gennes, The Physics of Liquid Crystals (Oxford University Press, New York, 1974).

${ }^{2}$ G. R. Luckhurst and G. W. Gray, Molecular Physics of Liquid Crystals (Academic Press, London, 1979).

${ }^{3}$ L. Onsager, Ann. N. Y. Acad. Sci. 51, 627-659 (1949).

${ }^{4}$ M. Franco-Melgar, A. J. Haslam, and G. Jackson, Mol. Phys. 106, 649-678 (2008).

${ }^{5}$ D. Frenkel and R. Eppenga, Phys. Rev. Lett. 49, 1089-1092 (1982).

${ }^{6}$ R. Eppenga and D. Frenkel, Mol. Phys. 52, 1303-1334 (1984).

${ }^{7}$ D. Frenkel, B. M. Mulder, and J. P. McTague, Phys. Rev. Lett. 52, 287-290 (1984).

${ }^{8}$ D. Frenkel and B. M. Mulder, Mol. Phys. 55, 1171-1192 (1985).

${ }^{9}$ A. Stroobants, H. N. W. Lekkerkerker, and D. Frenkel, Phys. Rev. Lett. 57, 1452-1455 (1986).

${ }^{10}$ A. Stroobants, H. N. W. Lekkerkerker, and D. Frenkel, Phys. Rev. A 36, 2929-2945 (1987).

${ }^{11}$ D. Frenkel, H. N. W. Lekkerkerker, and A. Stroobants, Nature 332, 822-823 (1988).

${ }^{12}$ D. Frenkel, J. Phys. Chem. 92, 3280-3284 (1988).

${ }^{13}$ J. A. C. Veerman and D. Frenkel, Phys. Rev. A 41, 3237-3244 (1990).

${ }^{14}$ M. P. Allen, Liq. Cryst. 8, 499-511 (1990).

${ }^{15}$ J. A. C. Veerman and D. Frenkel, Phys. Rev. A 45, 5632-5648 (1992).

${ }^{16}$ A. Samborski, G. T. Evans, C. P. Mason, and M. P. Allen, Mol. Phys. 81, 263-276 (1994).

${ }^{17}$ P. Bolhuis and D. Frenkel, J. Chem. Phys. 106, 666-687 (1997).

${ }^{18}$ D. C. Williamson and G. Jackson, J. Chem. Phys. 108, 10294-10302 (1998).

${ }^{19}$ A. Yethiraj and H. Fynewever, Mol. Phys. 93, 693-701 (1998).

${ }^{20}$ C. Vega, C. McBride, and L. G. MacDowell, J. Chem. Phys. 115, 4203-4211 (2001).

${ }^{21}$ B. Oyarzún, T. van Westen, and T. J. H. Vlugt, J. Chem. Phys. 138, 204905 (2013).

${ }^{22}$ T. van Westen, B. Oyarzún, T. J. H. Vlugt, and J. Gross, J. Chem. Phys. 139, 034505 (2013).

${ }^{23}$ T. van Westen, T. J. H. Vlugt, and J. Gross, J. Chem. Phys. 140, 034504 (2014).

${ }^{24}$ H. Finkelmann and H. J. Wendorff, Polym. Sci. Technol. 28, 295-302 (1985),

${ }^{25}$ T. van Westen, B. Oyarzún, T. J. H. Vlugt, and J. Gross, Mol. Phys. 112, 919-928 (2014).

${ }^{26}$ A. Z. Panagiotopoulos, Mol. Phys. 61, 813-826 (1987).

${ }^{27}$ A. Z. Panagiotopoulos, N. Quirke, M. Stapleton, and D. J. Tildesley, Mol. Phys. 63, 527-545 (1988).

${ }^{28}$ A. Z. Panagiotopoulos, Mol. Phys. 100, 237-246 (2002).

${ }^{29}$ A. Z. Panagiotopoulos, Fluid Phase Equilib. 76, 97-112 (1992).

${ }^{30}$ D. Frenkel, G. C. A. M. Mooij, and B. Smit, J. Phys.: Condens. Matter 4, 3053 (1992).

${ }^{31}$ J. I. Siepmann and D. Frenkel, Mol. Phys. 75, 59-70 (1992).

${ }^{32}$ J. J. de Pablo, M. Laso, and U. W. Suter, J. Chem. Phys. 96, 6157-6162 (1992).

${ }^{33}$ M. Laso, J. J. de Pablo, and U. W. Suter, J. Chem. Phys. 97, 2817-2819 (1992).

${ }^{34}$ J. I. Siepmann, S. Karaborni, and B. Smit, J. Am. Chem. Soc. 115, 6454-6455 (1993).

${ }^{35}$ J. I. Siepmann, S. Karaborni, and B. Smit, Nature 365, 330-332 (1993).

${ }^{36}$ B. Smit, S. Karaborni, and J. I. Siepmann, J. Chem. Phys. 102, 2126-2140 (1995).

${ }^{37}$ S. T. Cui, P. T. Cummings, and H. D. Cochran, Fluid Phase Equilib. 141, 45-61 (1997).

${ }^{38}$ J. I. Siepmann and M. G. Martin, Mol. Phys. 90, 687-694 (1997).

${ }^{39}$ T. J. H. Vlugt, M. G. Martin, B. Smit, J. I. Siepmann, and R. Krishna, Mol. Phys. 94, 727-733 (1998).

${ }^{40}$ T. J. H. Vlugt, Mol. Simul. 23, 63-78 (1999).

${ }^{41}$ S. Consta, T. J. H. Vlugt, J. W. Hoeth, B. Smit, and D. Frenkel, Mol. Phys. 97, 1243-1254 (1999).

${ }^{42}$ S. Consta, N. B. Wilding, D. Frenkel, and Z. Alexandrowicz, J. Chem. Phys. 110, 3220-3228 (1999).

${ }^{43}$ A. P. Lyubartsev, A. A. Martsinovski, S. V. Shevkunov, and P. N. VorontsovVelyaminov, J. Chem. Phys. 96, 1776-1783 (1992).

${ }^{44}$ N. B. Wilding and M. Müller, J. Chem. Phys. 101, 4324-4330 (1994).

${ }^{45}$ F. A. Escobedo and J. J. de Pablo, J. Chem. Phys. 103, 2703-2710 (1995).

${ }^{46}$ F. A. Escobedo and J. J. de Pablo, J. Chem. Phys. 105, 4391-4394 (1996).

${ }^{47}$ W. Shi and E. J. Maginn, J. Comput. Chem. 29, 2520-2530 (2008). 
${ }^{48}$ N. Metropolis, A. W. Rosenbluth, M. N. Rosenbluth, A. H. Teller, and E. Teller, J. Chem. Phys. 21, 1087-1092 (1953).

${ }^{49}$ B. A. Berg and T. Neuhaus, Phys. Lett. B 267, 249-253 (1991).

${ }^{50}$ N. B. Wilding, Am. J. Phys. 69, 1147-1155 (2001).

${ }^{51}$ C. R. A. Abreu and F. A. Escobedo, J. Chem. Phys. 124, 054116 (2006).

${ }^{52}$ A. Z. Panagiotopoulos, Mol. Simul. 9, 1-23 (1992).

${ }^{53}$ A. P. Lyubartsev, A. Laaksonen, and P. N. Vorontsov-Velyaminov, Mol. Phys. 82, 455-471 (1994).

${ }^{54}$ A. P. Lyubartsev, A. Laaksonen, and P. N. Vorontsov-velyaminov, Mol. Simul. 18, 43-58 (1996).

${ }^{55}$ W. Shi and E. J. Maginn, J. Chem. Theory Comput. 3, 1451-1463 (2007).

${ }^{56}$ A. Torres-Knoop, S. P. Balaji, T. J. H. Vlugt, and D. Dubbeldam, J. Chem. Theory Comput. 10, 942-952 (2014).

${ }^{57}$ F. A. Escobedo, J. Chem. Phys. 127, 174104 (2007).

${ }^{58}$ F. Wang and D. P. Landau, Phys. Rev. Lett. 86, 2050-2053 (2001).

${ }^{59}$ F. Wang and D. P. Landau, Phys. Rev. E 64, 056101 (2001).

${ }^{60}$ D. Frenkel and B. Smit, Understanding Molecular Simulations, 2nd ed. (Academic Press, London, 2002).

${ }^{61}$ M. G. Martin and J. I. Siepmann, J. Phys. Chem. B 102, 2569-2577 (1998).

${ }^{62}$ C. M. Wijmans, B. Smit, and R. D. Groot, J. Chem. Phys. 114, 7644-7654 (2001).

${ }^{63}$ M. S. Shell, P. G. Debenedetti, and A. Z. Panagiotopoulos, Phys. Rev. E 66, 056703 (2002).

${ }^{64}$ K. S. Shing and K. E. Gubbins, Mol. Phys. 46, 1109-1128 (1982).

${ }^{65}$ B. Widom, J. Chem. Phys. 39, 2808-2812 (1963).

${ }^{66}$ J. K. Shah and E. J. Maginn, J. Phys. Chem. B 109, 10395-10405 (2005).

${ }^{67}$ L. E. S. de Souza, A. Stamatopoulou, and D. Ben-Amotz, J. Chem. Phys. 100, 1456-1459 (1994).

${ }^{68}$ F. A. Escobedo and J. J. de Pablo, J. Chem. Phys. 106, 9858-9868 (1997).
${ }^{69}$ M. Adams, Z. Dogic, S. L. Keller, and S. Fraden, Nature 393, 349-352 (1998).

${ }^{70}$ H. N. W. Lekkerkerker and A. Stroobants, Nature 393, 305-307 (1998).

${ }^{71}$ D. Frenkel, J. Phys. chem. 91, 4912-4916 (1987).

${ }^{72}$ T. Odijk and H. N. W. Lekkerkerker, J. Phys. chem. 89, 2090-2096 (1985).

${ }^{73}$ S. Varga, A. Galindo, and G. Jackson, Mol. Phys. 101, 817-825 (2003).

${ }^{74}$ D. C. Williamson and G. Jackson, Mol. Phys. 86, 819-836 (1995).

${ }^{75}$ H. N. W. Lekkerkerker, Ph. Coulon, R. Van Der Haegen, and R. Deblieck, J. Chem. Phys. 80, 3427-3433 (1984).

${ }^{76}$ T. Odijk, Macromolecules 19, 2313-2329 (1986).

${ }^{77}$ G. Cinacchi, L. Mederos, and E. Velasco, J. Chem. Phys. 121, 3854-3863 (2004).

${ }^{78}$ G. Cinacchi, E. Velasco, and L. Mederos, J. Phys.: Condens. Matter 16, S2003 (2004).

${ }^{79}$ G. Cinacchi, Y. Martínez-Ratón, L. Mederos, and E. Velasco, Mol. Cryst. Liq. Cryst. 465, 121-132 (2007)

${ }^{80}$ G. Cinacchi, Y. Martínez-Ratón, L. Mederos, and E. Velasco, J. Chem. Phys. 124, 234904 (2006).

${ }^{81}$ T. van Westen, T. J. H. Vlugt, and J. Gross, J. Chem. Phys. 137, 044906 (2012).

${ }^{82}$ M. de Groen, T. J. H. Vlugt, and T. W. de Loos, J. Phys. Chem. B 116, $9101-$ 9106 (2012).

${ }^{83}$ M. de Groen, C. Ramaker, T. J. H. Vlugt, and T. W. de Loos, J. Chem. Eng. Data 59, 1667-1672 (2014).

${ }^{84}$ M. de Groen, T. J. H. Vlugt, and T. W. de Loos, "Binary and ternary mixtures with $\mathrm{CO}_{2}$," AIChE J. (submitted).

${ }^{85}$ D. G. Green, G. Jackson, E. de Miguel, and L. F. Rull, J. Chem. Phys. 101, 3190-3204 (1994).

${ }^{86}$ L. F. Rull, G. Jackson, and B. Smit, Mol. Phys. 85, 435-447 (1995). 